Abstract

\title{
Edge Modes in the Switching Mechanism of Finite Chains of Macrospins ${ }^{\dagger}$
}

\author{
Dominika Kuźma 1,*, Justyna Rychły ${ }^{2}$, Paweł Sobieszczyk ${ }^{1}$,Jarosław W. Kłos ${ }^{2}$, \\ Federico Montoncello ${ }^{3}$ and Piotr Zieliński ${ }^{1}$ \\ 1 Institute of Nuclear Physics Polish Academy of Sciences, PL-31342 Kraków, Poland \\ 2 Faculty of Physics, Adam Mickiewicz University, PL-61614 Poznań, Poland \\ 3 Dipartimento di Fisica e Scienze della Terra, Università di Ferrara, I-44122 Ferrara, Italy \\ * Correspondence: dominika.kuzma@ifj.edu.pl \\ + Presented at the 37th International Symposium on Dynamical Properties of Solids (DyProSo 2019), Ferrara, \\ Italy, 8-12 September 2019.
}

Published: 5 September 2019

We study transitions of $1 \mathrm{D}$ systems of macrospins between their equilibrium configurations under a uniform magnetic field and under variations of the distances of the macrospins in such chains. A magnetic field opens a gap at the Brillouin zone border, giving room to bound edge states. If the number of macrospins in the chain is odd, two bound states, symmetric and antisymmetric, appear in the gap for the magnetic field parallel to the magnetization of the end macrospins and the instability is driven by a bulk soft mode. If the field is oriented antiparallel to the end macrospins no bound modes appear in the gap. In turn the instability involves softening of edge bound states as illustrated in Fig.1. The even-odd alternation of this behaviour will be presented, as well as the effect of variations of the macrospins mutual distances.

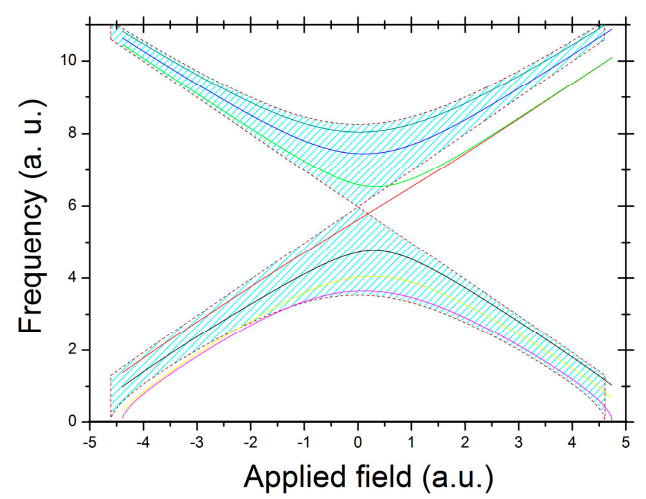

Figure 1. Bulk and bound states in a chain of seven macrospins in Stoner-Wohlfahrt model.

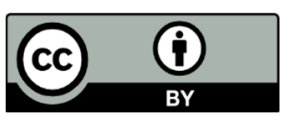

(C) 2019 by the authors. Licensee MDPI, Basel, Switzerland. This article is an open access article distributed under the terms and conditions of the Creative Commons Attribution (CC BY) license (http://creativecommons.org/licenses/by/4.0/). 